\title{
Reducing Noise Through Awareness in the NICU
}

Weis J, Haaber J

Rigshospitalet - Copenhagen University Hospital, Copenhagen, Denmark

\section{Aims/Purpose}

In the NICU, environment sounds and noise can be challenging for the preterm and/or sick newborn baby. Reducing noise to provide an environment with appropriate and meaningful auditory experiences such as parents' voices is important. Elimination of loud noises will furthermore benefit the families and staff. In 2016, the NICU at Rigshospitalet - Copenhagen University Hospital, collaborated with SoundEar ${ }^{\mathrm{TM}}$, a company that develops noise-meters for indicating and collecting noise levels, to develop a software program that was appropriate and easy to use. The aim was to support reduction in noise levels at the NICU through different layers of nudging: The noise meters with displays should help staff and families become aware of their own noise levels and change their noisy behavior. The software helps staff become aware of when and where noise levels are critical and something should be done differently. The software sends out noise reports on a weekly basis via email to key staff members, who use these reports as a basis for further discussion about noise at staff meetings.

\section{Methods}

Inspired by participatory research methods, SoundEar co-created the software program for hospital use with a group of staff from the NICU. Noise-meters were installed in all rooms. Half of the noise meters were anonymous white boxes, which solely measure and collect noise levels. The other half of the noise meters also have a display with an ear, that lights up green, yellow or red, indicating the current level of noise in the room. All the noise meters transfer noise measurement data wirelessly to a central computer, where it is accessible to staff through software. After having the SoundEar devices in the NICU for a few months, questionnaires about the perceived unit noise levels, and whether the SoundEar devices seemed to have changed anything, were distributed among the staff.

\section{Results/Findings}

It was determined that only a few staff members should be responsible for driving to the hospital to measure noise reduction rather than all staff members. Software-generated data was used to gather insights for the staff to discuss at weekly meetings. These insights turned into auto-generated noise reports being sent to key staff members to be discussed with staff.

Fourteen staff members, primarily nurses, answered the questionnaire, with $78.6 \%$ reporting that the SoundEar devices had made them more attentive to noise levels. The same amount reported to have changed some of their behaviour because of the SoundEar devices. The change that most staff members reported to have made, was when unpacking syringes and other types of medical equipment outside of patient rooms, because they no- ticed that the ripping of plastic made unnecessary noise around the babies. Others reported lowering their voices and lowering the noise level of alarms as changes they had made after the installation of the SoundEar devices. Several staff members also reported to have seen an increase in parents' attention to noise levels, and that they commented on noise to other visitors and siblings, thereby spreading the attention to noise.

\section{Conclusion}

A useful software program was developed to help strive to reduce unnecessary noise and promote appropriate surroundings in the NICU. Generation of daily or weekly reports of sound levels in each room may be used to promote and evaluate targeted noisereduction activities.

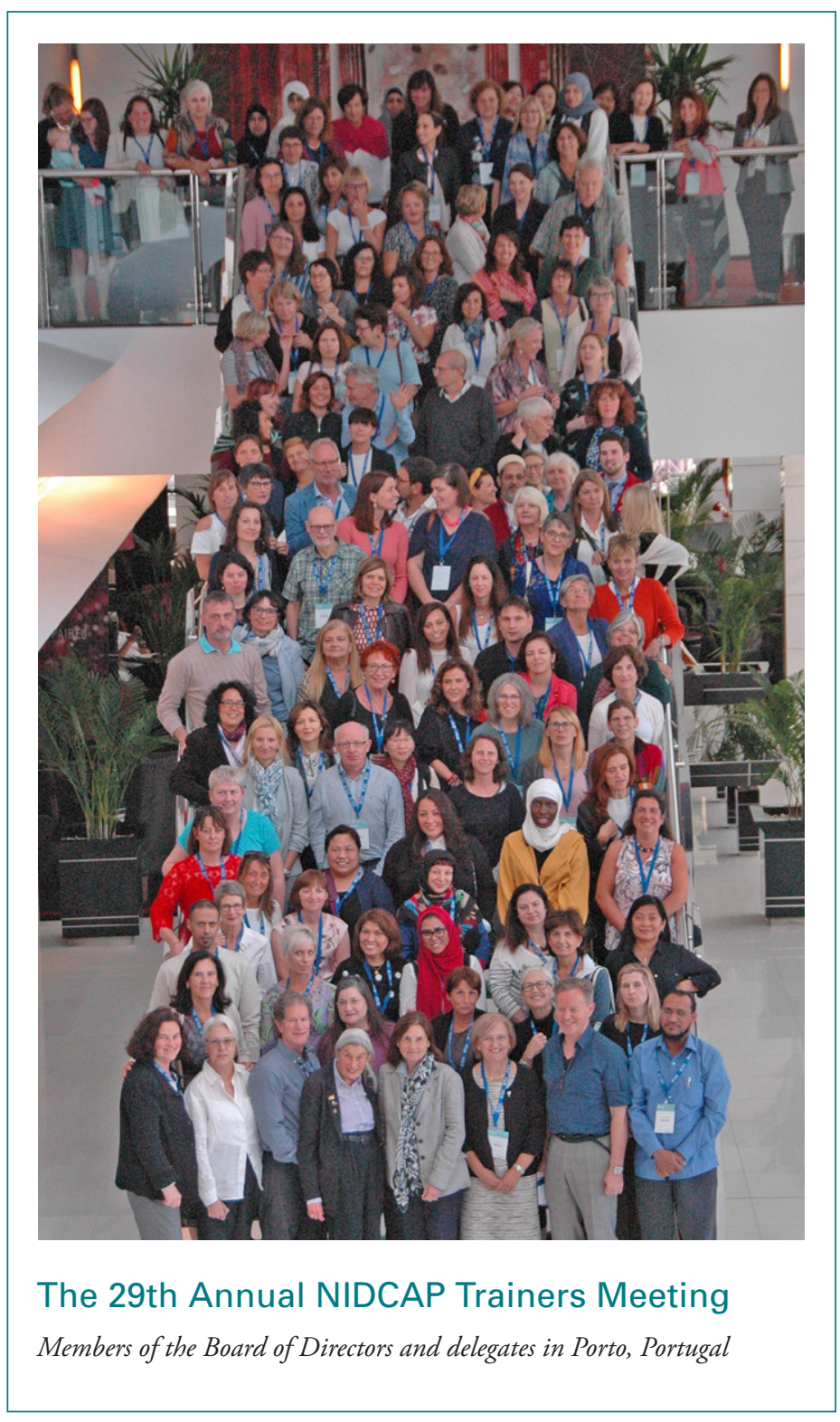

